\title{
Prototyping for the Spectropolarimeter for Planetary EXploration (SPEX): calibration and sky measurements
}

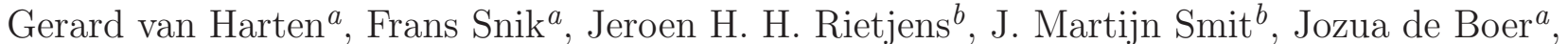 \\ Renia Diamantopoulou ${ }^{a}$, Otto P. Hasekamp ${ }^{b}$, Daphne M. Stam ${ }^{b}$, Christoph U. Keller ${ }^{a}$, Erik C. \\ Laan $^{c}$, Ad L. Verlaan ${ }^{c}$, Willem A. Vliegenthart ${ }^{c}$, Rik ter Horst ${ }^{d}$, Ramón Navarro ${ }^{d}$, Klaas

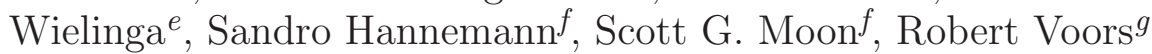

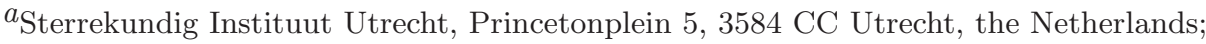 \\ ${ }^{b}$ SRON Netherlands Institute for Space Research, Sorbonnelaan 2, 3584 CA Utrecht, the Netherlands;

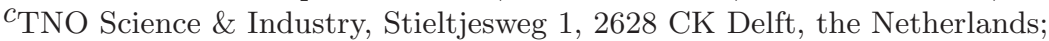

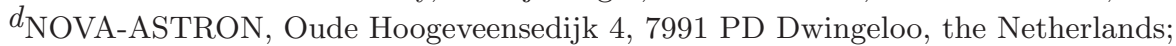

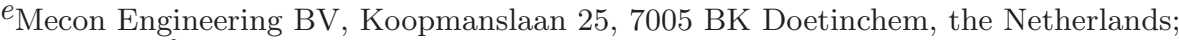

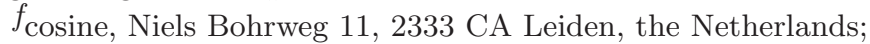

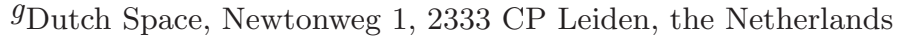

\begin{abstract}
We present the Spectropolarimeter for Planetary EXploration (SPEX), a high-accuracy linear spectropolarimeter measuring from 400 to $800 \mathrm{~nm}$ (with $2 \mathrm{~nm}$ intensity resolution), that is compact ( $\sim 1$ liter), robust and lightweight. This is achieved by employing the unconventional spectral polarization modulation technique, optimized for linear polarimetry. The polarization modulator consists of an achromatic quarter-wave retarder and a multiple-order retarder, followed by a polarizing beamsplitter, such that the incoming polarization state is encoded as a sinusoidal modulation in the intensity spectrum, where the amplitude scales with the degree of linear polarization, and the phase is determined by the angle of linear polarization. An optimized combination of birefringent crystals creates an athermal multiple-order retarder, with a uniform retardance across the field of view. Based on these specifications, SPEX is an ideal, passive remote sensing instrument for characterizing planetary atmospheres from an orbiting, air-borne or ground-based platform. By measuring the intensity and polarization spectra of sunlight that is scattered in the planetary atmosphere as a function of the single scattering angle, aerosol microphysical properties (size, shape, composition), vertical distribution and optical thickness can be derived. Such information is essential to fully understand the climate of a planet. A functional SPEX prototype has been developed and calibrated, showing excellent agreement with end-to-end performance simulations. Calibration tests show that the precision of the polarization measurements is at least $2 \cdot 10^{-4}$. We performed multi-angle spectropolarimetric measurements of the Earth's atmosphere from the ground in conjunction with one of AERONET's sun photometers. Several applications exist for SPEX throughout the solar system, a.o. in orbit around Mars, Jupiter and the Earth, and SPEX can also be part of a ground-based aerosol monitoring network.
\end{abstract}

Keywords: planet, atmosphere, aerosol, dust, Earth, Mars, remote sensing, spectropolarimetry, spectral modulation

\section{INTRODUCTION}

Atmospheric aerosol research is gaining more and more attention, mainly because of climate models showing their large impact on the Earth's climate, whereas the actual aerosol input for the models is poorly known [1]. Aerosols, $0.2-200 \mu \mathrm{m}$ sized particles suspended in the atmosphere, such as tiny water droplets, sand and sea salt, have a direct effect on the climate by absorbing and reflecting incoming sunlight, thereby cooling our planet. Moreover, some types of aerosols act as cloud condensation nuclei, with an increase of such aerosol leading

Further author information: (Send correspondence to G.v.H or F.S.)

G.v.H.: E-mail: g.vanharten@astro-uu.nl

F.S.: E-mail: f.snik@astro-uu.nl

Polarization Science and Remote Sensing V, edited by Joseph A. Shaw, J. Scott Tyo,

Proc. of SPIE Vol. 8160, 81600Z · (C) 2011 SPIE · CCC code: 0277-786X/11/\$18 · doi: 10.1117/12.893741

Proc. of SPIE Vol. $816081600 Z-1$ 
to clouds containing more and smaller water droplets. Since small droplets yield less efficient precipitation, they increase the lifetime of clouds, and hence influence the effects of clouds on the climate. The direct and indirect climate effects of aerosol particles are shown to be extremely sensitive to the aerosol microphysical properties, such as size, shape and chemical composition, and to their concentration and spatial distribution. Hence, accurate aerosol characterization is necessary for a thorough understanding of our climate, and it may ultimately give us a mechanism for climate control, by regulating the anthropogenic aerosol contribution, such as combustion soot and smoke, but also artificial nanoparticles. Clearly, continuous monitoring of atmospheric aerosol on small spatial and time scales is important because of their immediate and local effects on, e.g., air quality and health. Moreover, a monitoring system can minimize the direct economical impact of, e.g., volcanic ash clouds, hampering air traffic.

By studying different planetary atmospheres, such as Mars, Jupiter, Saturn and Venus, each having a completely different climate, we deepen our knowledge about climate systems in general. In addition, it allows for studying individual phenomena, such as the extreme dust storms on Mars, thereby giving insight into elements of our own climate, which cannot be studied separately in the Earth's complex atmosphere.

Obviously, remote sensing is the way to obtain the required coverage, either from an airborne platform (e.g. from a satellite, balloon or airplane(s)), or from a ground-based network. By measuring the spectral intensity and linear polarization of sunlight, scattered at different angles by a patch of atmosphere, and by fitting this to radiative transfer calculations, the micro- and macrophysical aerosol properties can be determined unambiguously $[2,3]$. This powerful technique has already been used in several missions, such as Pioneers 10, 11 and Venus, Voyagers 1 and 2, Galileo, and in various Earth observing missions using the POLDER polarimeter. So far, polarimetry has only been performed using either temporal or spatial polarization modulation, which have their intrinsic problems [see 4]. Temporal modulation implies the measurement of the input polarization by applying different modulation states sequentially. However, during a sequence, the polarimeter is looking at different scenes due to the movement of the platform, thereby inducing spurious polarization signals. For spatial modulation, the beam is split up, such that different modulation states can be measured simultaneously. The difference in the beams' optical paths again induces spurious polarization.

We present SPEX (Spectropolarimeter for Planetary EXploration), which employs a novel spectral polarization modulation technique, providing high-accuracy linear spectropolarimetry at moderate spectral resolution throughout the visible, at the same time being fully passive, compact, robust and lightweight. The instrument principle is explained in Section 2. A functional prototype has been developed, which design is shown in Section 3. The first results obtained with this prototype are presented in Section 4, viz. several calibration results, as well as ground-based measurements of aerosols in the Earth's atmosphere. Section 5 describes the future perspective of SPEX, including the current efforts being taken to be ready for missions to Mars and Jupiter, as well as for Earth observation from space and from the ground.

\section{SPEX INSTRUMENT PRINCIPLE}

SPEX is a fully passive high-accuracy linear spectropolarimeter with moderate spectral resolution throughout the visible, that is compact $(\sim 1$ liter $)$, robust and lightweight. This is achieved by employing the unconventional spectral polarization modulation technique, also known as channeled spectropolarimetry, optimized for linear polarimetry [see 5]. The polarization modulator consists of an achromatic quarter-wave retarder and a multipleorder retarder, followed by a polarizing beamsplitter, such that the incoming polarization state is encoded as a sinusoidal modulation in the intensity spectrum, where the amplitude scales with the degree of linear polarization (DoLP), and the phase determines the angle of linear polarization (AoLP) (see Fig. 2). It can be shown that the modulated spectrum, as a function of the incoming intensity spectrum $I_{0}(\lambda)$, the incoming linear polarization $\operatorname{DoLP}(\lambda)$ and $\operatorname{AoLP}(\lambda)$, and the waveplate retardance $\delta(\lambda, T)$, can be written as

$$
I_{ \pm}(\lambda)=\frac{1}{2} I_{0}(\lambda)\left[1 \pm \operatorname{DoLP}(\lambda) \cos \left(\frac{2 \pi \delta(\lambda, T)}{\lambda}+2 \operatorname{AoLP}(\lambda)\right)\right]
$$

where the \pm sign corresponds to the analyzer being parallel or perpendicular to the quarter-wave retarder. 


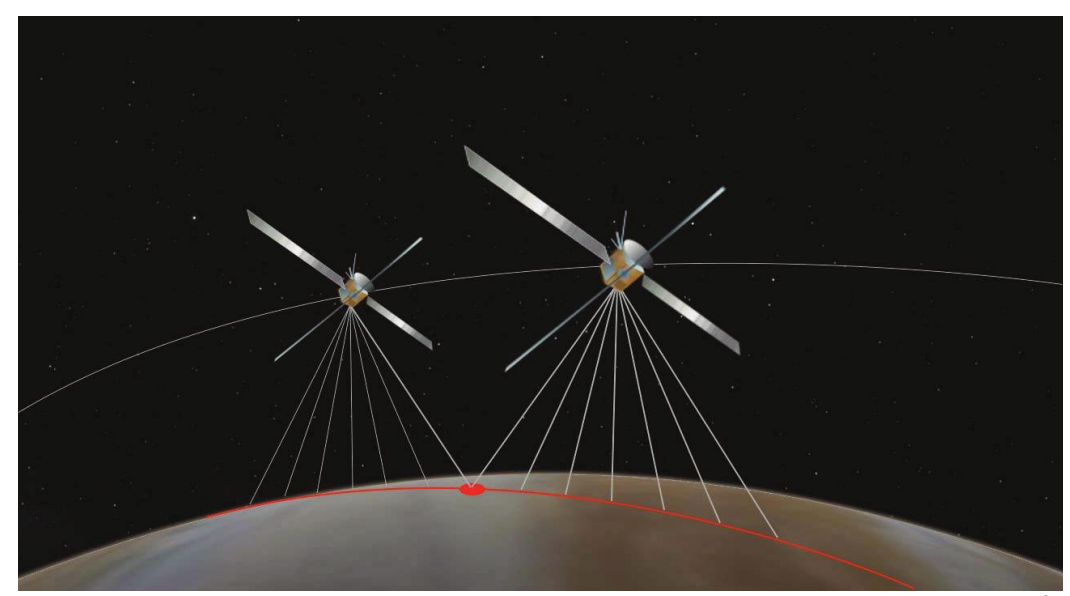

Figure 1: SPEX onboard a satellite measures the intensity and linear polarization of sunlight scattered in a planetary atmosphere, for 9 ground pixels along the track simultaneously. While flying over the planet, each ground pixel has eventually been observed at 9 different scattering angles. Combined with the moderate spectral resolution throughout the visible, the parameter space is sufficiently sampled to reveal the micro- and macrophysical properties of the atmospheric aerosol, by interpreting the measurements with radiative transfer calculations.

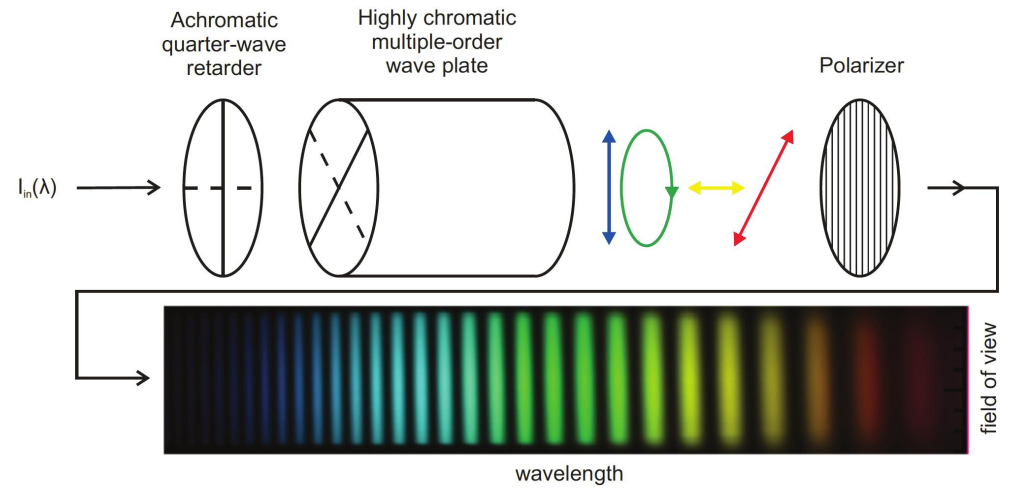

Figure 2: The SPEX principle, viz. the spectral modulation of linear polarization. The colored arrows after the highly chromatic multiple-order waveplate denote the strongly wavelength dependent polarization modulation. Analyzing this polarization leads to a characteristic intensity spectrum, containing the linear polarization information, according to equation (1).

A demodulation algorithm determines for every wavelength the local modulation amplitude $(\operatorname{DoLP}(\lambda))$ and phase $(\operatorname{AoLP}(\lambda))$, as well as the unmodulated intensity $\left(I_{0}(\lambda)\right)$, resulting in a spectral resolution of roughly a factor 10 worse than the sampling resolution. This is the price we have to pay for using spectral modulation, however, as long as the linear polarization varies slowly across the spectrum, which applies for scattering polarization, these data products can be achieved very accurately and with sufficient spectral resolution for aerosol characterization. Note that the period of the modulation is constant in the inverse wavelength domain, so for shorter wavelengths the modulations are faster and the spectral resolution is higher.

In case the analyzer is implemented as a polarizing beam splitter, both $I_{+}(\lambda)$ and $I_{-}(\lambda)$ are measured, and the sum of these spectra yields the unmodulated intensity spectrum at full spectral resolution. The issue of differential transmission can be circumvented by realizing that the normalized modulations, i.e. $I_{ \pm} / I_{0}$, should be centered around $1 / 2$. Systematic deviations from this value reveal the differential transmission, according:

$$
\frac{T_{+}}{T_{-}}(\lambda)=\left\langle\frac{I_{-}}{I_{+}+I_{-}}\right\rangle^{-1}(\lambda)-1
$$

where the operator \langle\rangle means spectral averaging of the argument, such that the modulation is eliminated. This 
way, differential transmission is deduced from the measured spectra themselves, in addition to separate calibration measurements.

The $T$ in equation (1) denotes the strong temperature dependence of the multiple-order retarder. The equation shows that temperature changes directly translate into apparent changes in the AoLP. In order to keep the instrument passive and energy efficient, temperature control is avoided, using the following solution: the retarder consists of two plates with opposite temperature effects. Measurements published in reference [7] show that a subtractive combination of $\mathrm{MgF}_{2}$ and $\mathrm{Al}_{2} \mathrm{O}_{3}$ (i.e. with their fast axes crossed), with a thickness ratio of $2.4: 1$, yields optimum performance over the wavelength range $350-800 \mathrm{~nm}$. The residual temperature effect is less than $1.5 \cdot 10^{-2} \mathrm{rad} /{ }^{\circ} \mathrm{C}$, and can be corrected during data reduction if the temperature is known, or the other way around, the actual temperature can be deduced if the incoming AoLP is known, which is the case for scattering polarization. Note that our main observable, the DoLP, is (to first order) insensitive to changes in the multiple-order retardance, e.g. due to temperature changes.

Although the SPEX principle is generic, the instrument can be optimized for specific applications. For example, different planets can have maximum atmospheric information in different wavelength ranges, and can require different spectral or spatial resolutions and coverage. Moreover, orbit specifications differ from mission to mission. SPEX has been submitted as scientific payload instrument for the Mars Trace Gas Mission orbiter, as part of the former ESA-NASA ExoMars Programme. At 300-500 km altitude, it would characterize airborne dust and soil, as well as ice clouds high in the atmosphere [6]. The corresponding specifications of SPEX are shown in Table 1, which also forms the baseline of the constructed prototype, as presented in the next sections.

\begin{tabular}{|l|l|}
\hline spectropolarimeter volume & $\sim 1 \mathrm{l}$ \\
\hline mass including electronics & $\sim 2 \mathrm{~kg}$ \\
\hline maximum power consumption & $\sim 2 \mathrm{~W}$ \\
\hline spectral range & $400-800 \mathrm{~nm}$ \\
\hline spectral resolution for polarization & $20 \mathrm{~nm}$ \\
\hline spectral resolution for intensity & $2 \mathrm{~nm}$ \\
\hline viewing directions & 9 along the track: $0, \pm 14, \pm 28, \pm 42, \pm 56^{\circ}$ \\
\hline field of view for each viewing direction & $1^{\circ} \times 7^{\circ}($ cross-flight, to deal with planet's rotation) \\
\hline pupil size for each viewing direction & $\sim 1.1 \mathrm{~mm}^{2}$ \\
\hline measured polarization properties & Stokes $I, Q \& U$ \\
\hline polarization sensitivity & $0.005($ degree of linear polarization $)$ \\
\hline relative polarization accuracy & $5 \%($ down to 0.01 absolute $)$ \\
\hline
\end{tabular}

Table 1: Typical specifications for SPEX onboard the Mars Trace Gas Mission orbiter. From [7].

\section{PROTOTYPE DESIGN}

\subsection{OPTICAL DESIGN}

The optical system described below can be divided into two main parts; the pre-slit optics (which includes the polarimeters), and the spectrometer optics. Nine fields of view, of which each individual field is split according to two orthogonal polarization states, should be imaged within the sides of the $12.5 \times 12.5 \mathrm{~mm}, 512 \times 512$ pixels detector. For the SPEX prototype, only 3 fingers are filled with polarization optics. This section is largely based on the work in [7].

\subsubsection{Pre-slit Optics}

The incoming beam, which has a diameter of $1.1 \mathrm{~mm}$, will first pass the polarization optics to ensure zero instrumental polarization: a BK7G18 Fresnel rhomb for the achromatic quarter-wave retarder, an athermal combination of $1.22 \mathrm{~mm}$ sapphire and $2.88 \mathrm{~mm} \mathrm{MgF}$ for the thick retarder and an $\alpha \mathrm{BBO}$ Wollaston prism for the polarizing beam-splitter, see Fig. 3b. All optical components are non-moving. In the baseline design, only one single lenslet $(\mathrm{F}=10 \mathrm{~mm})$ focuses the two polarized beams on two separated slits $(1.4 \times 0.2 \mathrm{~mm})$. The advantage of this solution is that only nine relatively large lenses can be used (although still being just $4 \mathrm{~mm}$ in diameter), relaxing the manufacturing tolerances as compared to a solution using 18 smaller lenslets (one for each field and polarization state). The dispersion within the Wollaston prism is largely compensated by the dispersion caused by the off-axis light path through the lenslets. 


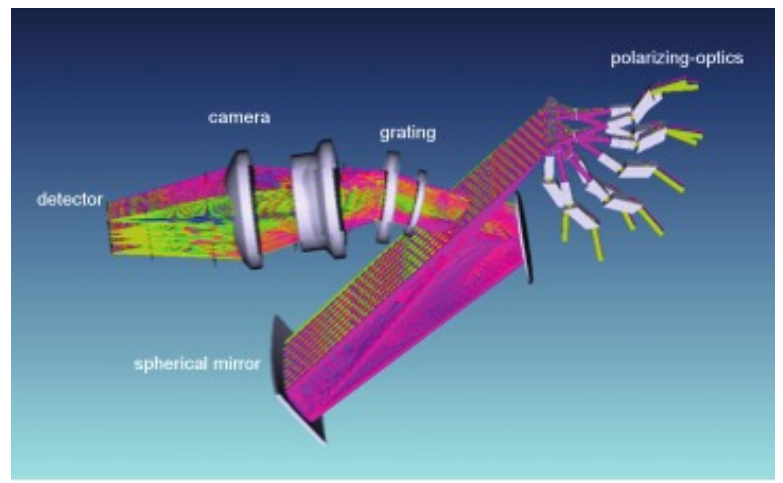

(a)

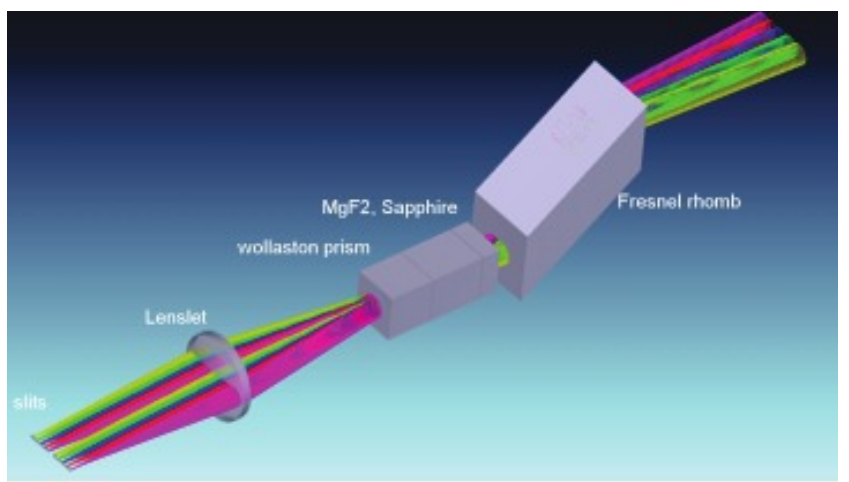

(b)

Figure 3: (a) Optical design for SPEX. Light enters the 9 fingers with polarization optics on the upper right, is directed to the slit plane via 9 mirrors (not shown), then goes through the spectrograph optics, onto the detector. For the prototype, only 3 fingers are filled with polarization optics, and the detector is an off-the-shelf camera, connected to the SPEX housing. (b) One of the fingers containing the polarization optics for one viewing direction. Light enters the finger on the upper right. From [7].

\subsubsection{Spectrometer Optics}

Light will enter the slit with a focal ratio of $\mathrm{F} / 10$, and will be demagnified by the camera lens into $\mathrm{F} / 3.3$ in order to have a good match with the detector and the specified spectral resolution. The current design has been optimized for an altitude of $300 \mathrm{~km}$, with viewing angles of $0, \pm 14, \pm 28, \pm 42, \pm 56$ degrees. The camera contains three radiation-resistant glass lenses of which two are identical aspheres (see Fig. 3a). Manufacturing aspherical surfaces is more difficult than spherical surfaces, but modern manufacturing techniques nowadays can guarantee good quality. Testing is more straightforward as only one test setup is needed to test the twin aspherical components, reducing the costs of such a set-up. For the SPEX prototype, the off-the-shelf CCD camera QImaging Retiga 4000R is used, which has an extremely low dark current of $1.64 \mathrm{e}^{-} / \mathrm{pix} / \mathrm{s}$, a read noise of $12 \mathrm{e}^{-}$, and a linear full well depth of $40,000 \mathrm{e}^{-}$.

For reasons of compactness we decided to use a (quartz) transmission grating, having a spacing of 750 lines per mm. Spectral overlap of orders is partially solved by the quantum efficiency of the intended detector, which is typically zero outside 400-1000 $\mathrm{nm}$, which means that together with the low transmission of the radiation resistant F2G12 lens at shorter wavelengths, the 350-400 nm regions will not be registered. Further filtering of the unwanted wavelengths $(750-1000 \mathrm{~nm})$ is done by using a blocking filter.

At least $80 \%$ of the energy of all fields and wavelengths should be focused within an area of $2 \times 2$ pixels $(50 \times 50$ $\mu \mathrm{m})$, which has been achieved with this optical design.

\subsection{MECHANICAL DESIGN}

Several renderings of the mechanical design of the SPEX prototype are shown in Fig. 4.

The SPEX prototype is designed for environmental testing of the instrument to allow for TRL6 qualification. As such the mechanical design incorporates many design features aimed at the operation of the instrument in harsh space environment and survival of the launch vibrations. In addition the design is very stiff, yet light (0.9 $\mathrm{kg})$ and compact ( $<1$ liter). The main frame is made out of a solid block of Aluminum of $12 \times 12 \times 6 \mathrm{~cm}$ into a structure of $\sim 1.5 \mathrm{~mm}$ thickness. Due to the combination of smart design and modern production techniques like spark eroding and diamond turning, we have succeeded in producing a design that requires no active alignment of optical elements, other than the focusing of the spectrometer detector.

A number of numerical simulations have been performed to verify and support the mechanical design. This comprises Finite Element Model (FEM) thermal analysis, stiffness and strength calculations. In particular a large effort was made to reduce the stresses exerted on the elements of the polarization optics. In general changes of stress on optical elements will result in variation of the birefringence. As a result the polarization measurement 


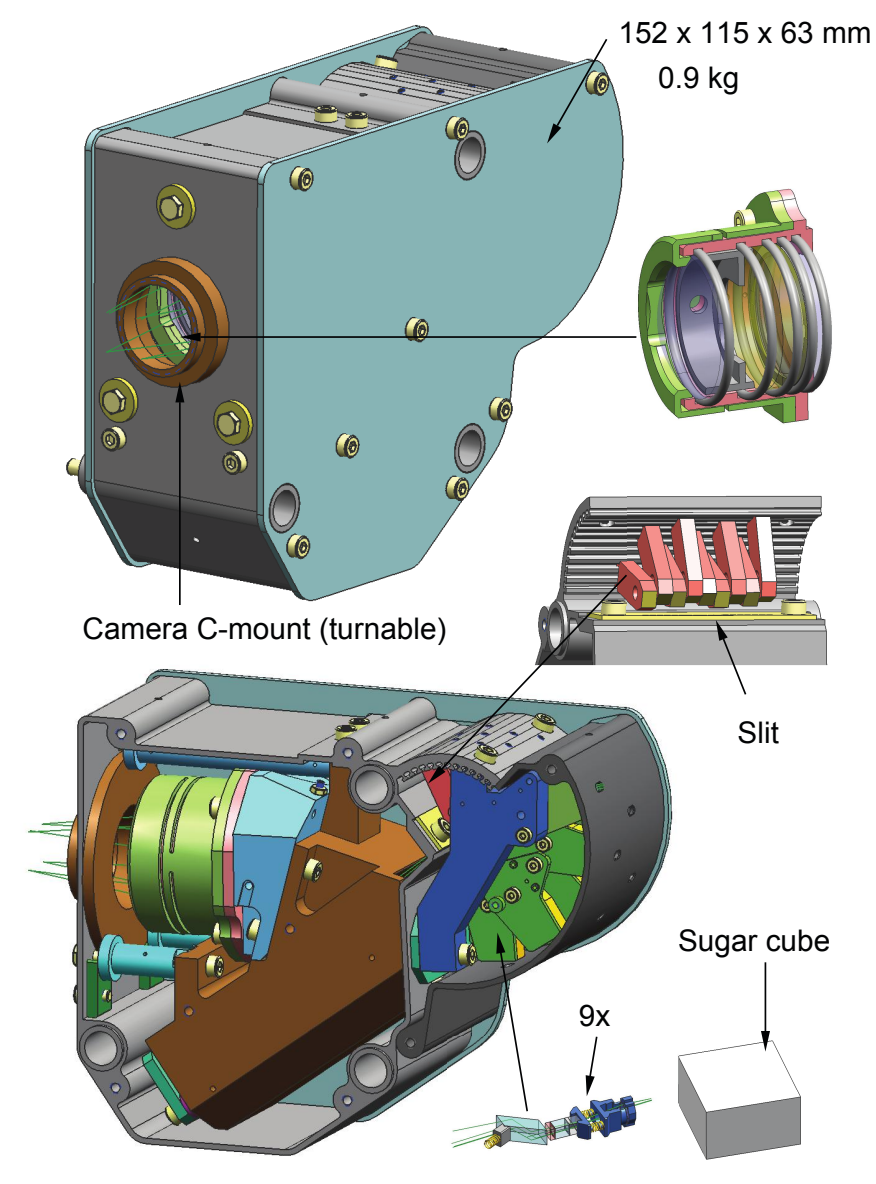

Figure 4: Multiple views of the mechanical design of the SPEX prototype.

would be affected in an unpredictable manner and after the calibration of the instrument. As a result, the optical elements are mounted very stiff to survive the launch vibrations, but yet with very low forces to minimize the induced stresses.

\section{PROTOTYPE RESULTS}

A prototype SPEX instrument has been constructed (see Fig. 5), based on the specifications as listed in Table 1, and by the design as presented in Section 3. Several calibration measurements are being performed to characterize its spectral- and field-of-view behavior, as well as its polarimetric performance. Although the calibration program is still ongoing, some promising results are already presented in Subsection 4.1. A detailed field-of-view characterization will be presented in a forthcoming paper, and we plan to determine the polarimetric accuracy within a year.

Ground-based aerosol measurements have been performed using the prototype, as presented in Subsection 4.2. 


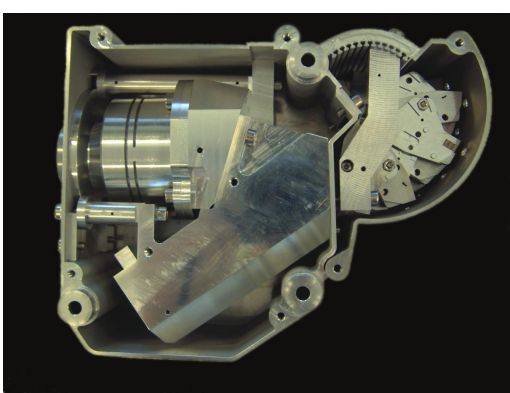

(a)

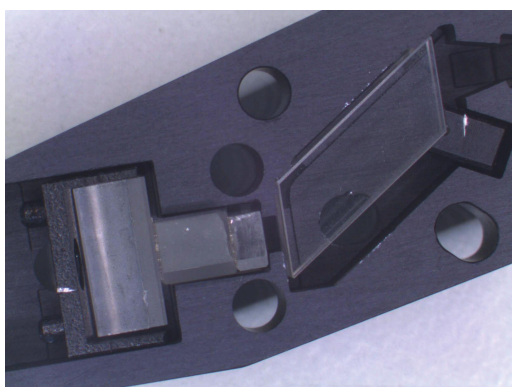

(b)

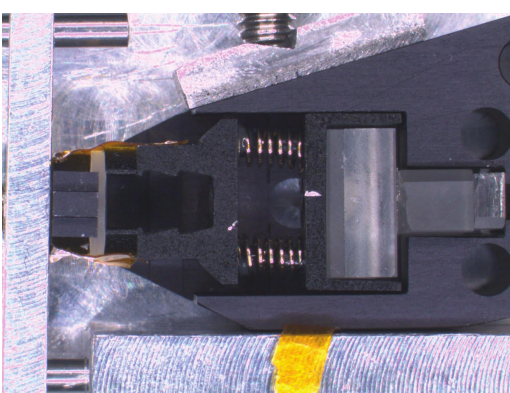

(c)

Figure 5: (a) The constructed SPEX prototype, with the top lid removed (detector is not shown). (b) Close-up on one of the fingers containing the polarization optics, viz. the BK7G18 Fresnel rhomb, $\mathrm{Al}_{2} \mathrm{O}_{3}$ crystal, $\mathrm{MgF}_{2}$ crystal, and $\alpha \mathrm{BBO}$ Wollaston prism, from right to left. (c) Another close-up on one of the fingers, showing the spring loaded polarization optics mount, the baffling and slit lens.

\subsection{CALIBRATION}

A raw detector image showing the two perpendicularly polarized spectra for each of the three fingers containing optics is shown in Fig. 6a. This image has been used to determine the pixels corresponding to the centra of the fields-of-view, which show a curvature from 400 to $800 \mathrm{~nm}$ of 10 pixels, away from the center of the detector, for the lower and upper finger, when the spectra of the central finger are perfectly horizontal.

The wavelength calibration has been performed by taking exposures of an $\mathrm{HgAr}$ spectral line lamp, which produces tens of emission lines between 250 and $920 \mathrm{~nm}$. First the isolines of six wavelengths, quite uniformly distributed between 400 and $800 \mathrm{~nm}$, are determined by fitting a quadratic function through the pixels corresponding to the centra of the fields-of-view, containing the maximum line intensities. Then a third order polynomial is fitted through each detector row, to obtain a complete wavelength map for the detector, as shown in Fig. 6b.

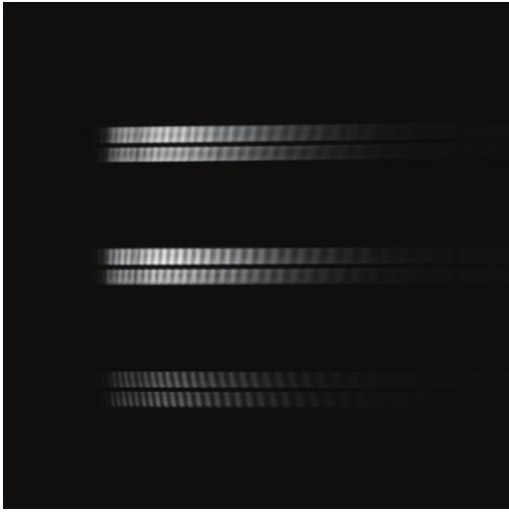

(a)

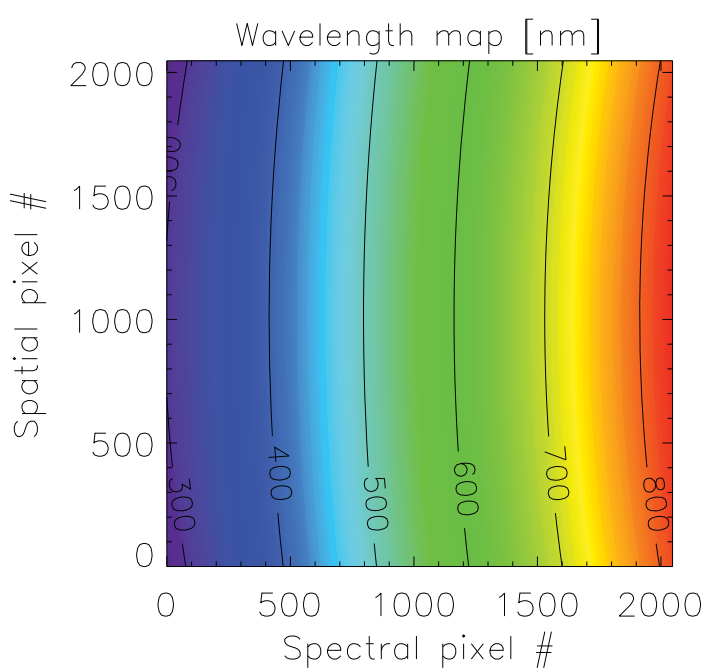

(b)

Figure 6: (a) Raw detector image of the SPEX prototype, with all three fingers filled with polarization optics illuminated. For each finger, two perpendicularly polarized spectra are measured, corresponding to the two output beams of the Wollaston analyzer. (b) The determined wavelength map, assigning a wavelength to each detector pixel.

The polarimetric efficiency of the central finger has been measured by placing a polarizer at different AoLPs in front of the SPEX prototype. The thus determined DoLP efficiency, averaged over $180^{\circ}$ AoLP and corrected 
for the polarizer's spectral polarizance, is strongly dependent on wavelength (see Fig. 7a), with a maximum efficiency of about $90 \%$. This is fully predicted by the SPEX instrument simulator [7], and can be attributed to the slit function of the spectrometer, in combination with spectral modulation, which is faster at the blue side of the spectrum, such that in the blue the measured intensity is an average over a larger part of the modulated input spectrum. In addition to this spectral dependence, the polarimetric efficiency is also a function of the AoLP of the incoming light, as shown in Fig. $7 \mathrm{~b}$, showing an amplitude of 0.005 for the efficiency variation. One would expect the highest polarimetric efficiency at AoLP $=0$, because Stokes $Q(+Q$ is in the plane of the Fresnel rhomb's internal reflecting surface) can simply pass through the Fresnel rhomb, whereas an imperfect conversion of $U$ into $V$ causes a decrease in efficiency. However, the highest observed efficiency is close to AoLP $=45^{\circ}$, which can be explained by a misalignment between the Fresnel rhomb and the multiple-order retarder. Stokes $Q$, after passing through the Fresnel rhomb, will then be partially converted into $V$, so that it is not fully transmitted or blocked by the analyzer, which is a decrease in $Q$ efficiency. Stokes $U$, however, will be converted into $V$, which is always fully converted into linear polarization, albeit at the same offset angle as the multiple-order retarder with respect to Stokes $U$. This effect apparently drowns out the imperfect Fresnel rhomb performance. Calculations show that, upon neglecting the Fresnel rhomb performance, a misalignment of $1.6^{\circ}$ can account for this efficiency variation of size 0.005 , which is in perfect agreement with the measured misalignment of $1.7^{\circ}$ (see Fig. $7 \mathrm{~b}$ ). The wavelength dependence of the efficiency curve follows from the dispersion of the Fresnel rhomb retardance, so eventually this efficiency curve should be determined also as a function of wavelength.

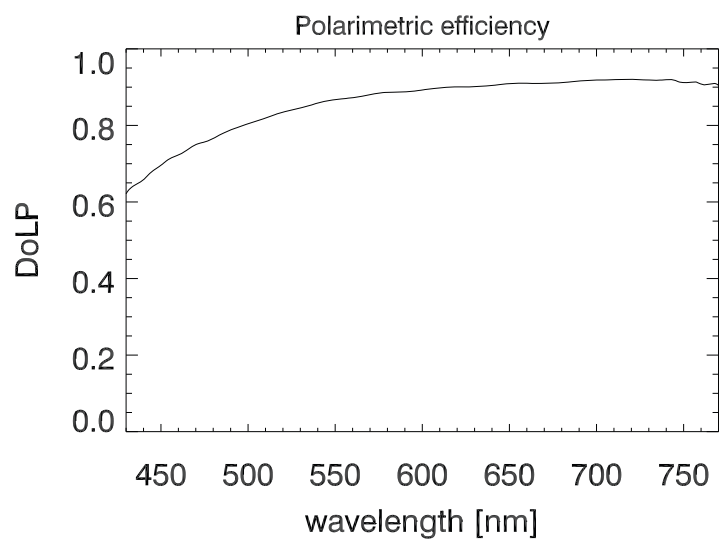

(a)

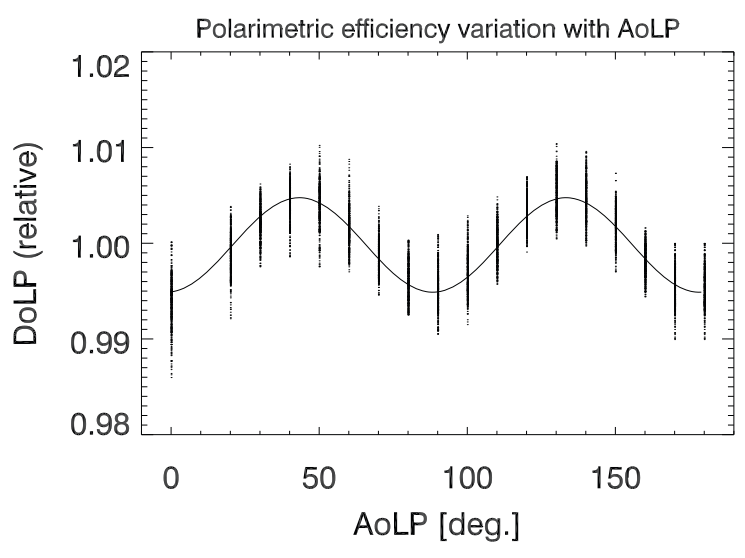

(b)

Figure 7: (a) The determined spectral polarimetric efficiency, i.e., the measured DoLP in case of 100\% linearly polarized input. (b) The measured variation on the polarimetric efficiency, as a function of the AoLP of the incoming light. The relative polarimetric efficiency is determined for all wavelengths simultaneously (dots). A fit of a sine function to the data yields an efficiency variation of 0.005 (solid line).

Partial polarization measurements have been performed, in order to assess the polarimetric performance for DoLP $<0.30$, which is the usual regime for scattering polarization measurements. The partial polarization states are created by transmitting light through a glassplate at different angles of incidence. The polarization of the incident light is unknown, as well as the refractive index of the glassplate, so the measurements are fitted to a Mueller matrix model containing these as free parameters. The measurements as a function of different angles of incidence are shown in Figs. 8a-c, and the fit residuals are shown in Figs. 8d-f. Although the fit residuals across the whole range of incident angles (see Fig. 8d) are already within \pm 0.006 , the (at least relative) accuracy is expected to be even better, because the systematic errors in the fit residuals indicate that the model is not complete. For example, the effect of multiple reflections inside the glass plate is neglected, as well as beam shift at large angles of incidence. Due to symmetry, the fit residuals for incidence angles close to 0 (see Fig. 8e) are almost free from systematic behavior as a function of incident angle, so these measurements can be used to determine the noise level: the total standard deviation, corrected for offsets between different wavelengths, is $4.8 \cdot 10^{-4}$, after averaging 16 almost saturated exposures. The pixels of the off-the-shelf detector of the prototype are 4 times smaller than necessary to meet the required spatial and spectral resolution, so the noise after binning $4 \times 4$ pixels is a more relevant quantity, which is $2.0 \cdot 10^{-4}$. 


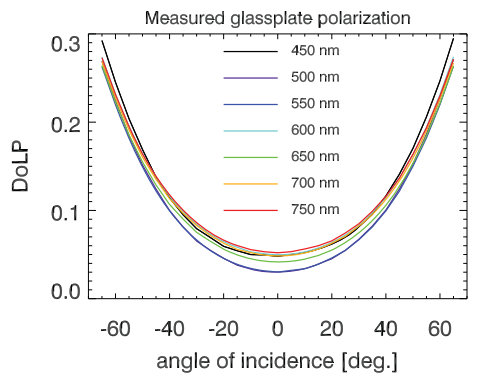

(a)

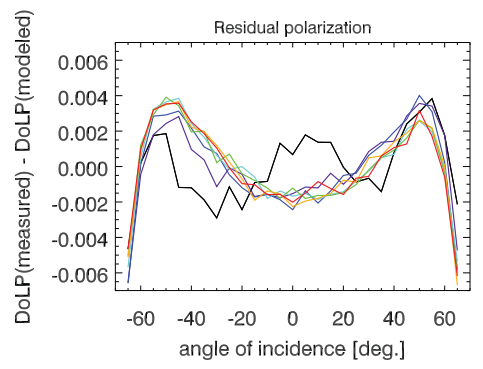

(d)

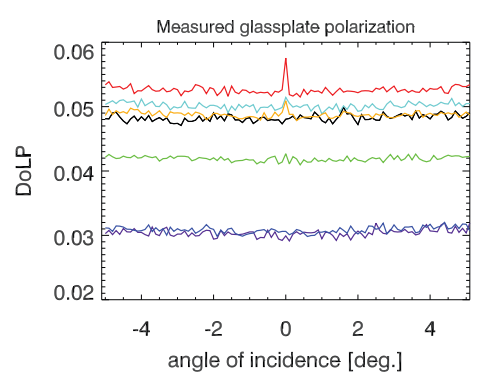

(b)

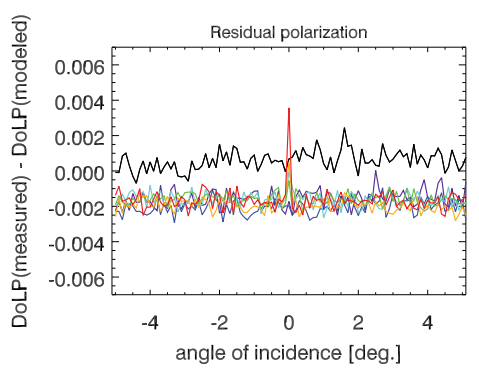

(e)

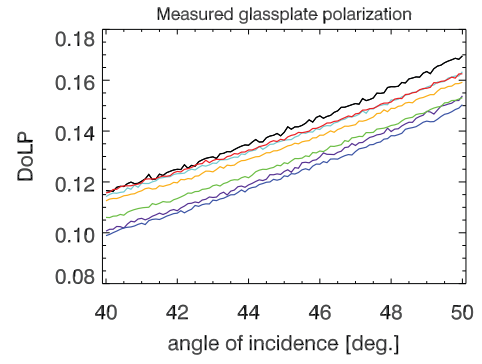

(c)

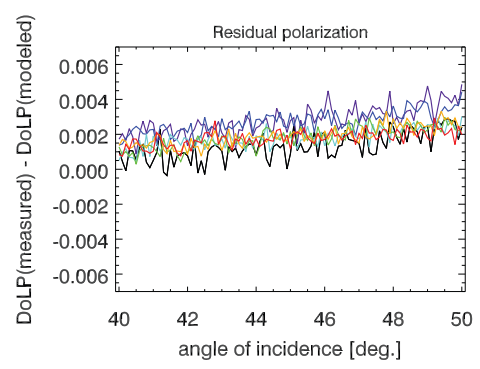

(f)

Figure 8: Polarization measurements by the SPEX prototype, where the partial polarization states are created by transmitting light through a glassplate at different tilt angles. (a) The full range of incidence angles is sampled with an angular resolution of $5^{\circ}$, and fine steps of $0.1^{\circ}$ are taken around (b) $0^{\circ}$ and (c) $45^{\circ}$ incidence. The polarization of the incident light is unknown, as well as the refractive index of the glassplate, so the measurements for the full range of incidence angles (subfigure (a)) are fitted to a Mueller matrix model containing these as free parameters. The measurements as a function of different glassplate tilt angles are shown in (a-c), and the fit residuals are shown in (d-f). The systematic errors in the fit residuals indicate that the model is not complete. The noise level in subfigure (e) is $4.8 \cdot 10^{-4}$, which becomes $2.0 \cdot 10^{-4}$ after binning $4 \times 4$ pixels, corresponding to the required spatial and spectral resolution.

\subsection{BLUE SKY MEASUREMENTS}

We performed polarization measurements from the ground with the SPEX prototype to verify its ability to determine atmospheric aerosol properties. The measurements are performed at the Cabauw Experimental Site for Atmospheric Research (CESAR, $51^{\circ} 58.223^{\prime} \mathrm{N}, 4^{\circ} 55.575^{\prime} \mathrm{E}$ ), where different aerosol instruments are located, such as an AERONET sun photometer, PM10 and PM2.5 filters and LIDARs, which can be used to either constrain the aerosol retrieval, or to verify retrieved parameter values. The site is located in a rural area, where the closest city is at a distance of 15 kilometers. Within 10 kilometers of the site, the surface is mainly covered with grass.

At three times during the cloudless day of May 25, 2011, the instrument was pointed at about 20 angles from horizon to horizon to sample the scattering phase function. As an example, the dataset corresponding to one of the three sweeps will be discussed now. The viewing angles are in the north-south plane, and the data was taken between 2:36 and 3:14 pm $(\mathrm{GMT}+2)$, so the Sun's altitude and azimuth are about $55^{\circ}$ and $215^{\circ}$, respectively. Data from all three viewing apertures containing optics has been collected, although here we present only the results for the central finger. Fig. 9a represents the viewing zenith angles of the measurements that are far enough from the Sun (depicted by the asterisk) to not be subject to possible straylight or detector saturation. The measured polarization as a function of single scattering angle for different wavelengths is shown in Fig. 9b, showing maximum values around a single scattering angle of $90^{\circ}$. The two outliers around the $50^{\circ}$ single scattering angle correspond to the two measurements close to the south horizon (see Fig. 9a), which have a completely different path through the atmosphere than the measurements at the same single scattering angle close to zenith, so the decreased DoLP may be due to multiple scattering. The complete dataset, viz. the measured spectral DoLP for the different viewing angles, is shown in Fig. 9c. The following features can be 
observed:

- The DoLP is relatively high at the shortest wavelengths, because there scattering by molecules, with a relatively high single scattering degree of polarization, plays a significant role. With increasing wavelength, the scattering optical thickness of the molecules decreases, and the polarization is increasingly determined by light that has been scattered by the aerosol particles.

- Around $550 \mathrm{~nm}$, the DoLP is relatively low because of the contribution of weakly polarized light that has been reflected towards the atmosphere by the surface (the so-called 'green bump'). At the Cabauw site, the surface is mainly covered by grass, which has a relatively high albedo at green wavelengths. The increased contribution of weakly polarized light reflected by the grass can also be seen above $700 \mathrm{~nm}$, since vegetation has high albedos at these long wavelengths (the so-called 'red edge').

See reference [9] for an extensive description of blue sky polarization measurements with the Global Ozone Monitoring Experiment Bread Board Model (GOME BBM) at 300-800 nm with a spectral resolution of about $0.3 \mathrm{~nm}$, and modeling thereof.

Although the polarimetric accuracy of the SPEX prototype for partial polarization measurements is yet to be investigated, the most reliable estimate of the accuracy after calibration so far is given by the wavelength dependent variation of the data in Fig. $7 \mathrm{~b}$, which is 0.005 . In the near future the aerosol retrieval will be performed, by first calculating a general look-up-table of spectral polarization as a function of all relevant aerosol parameters, for a large range of scattering angles, using an adding-doubling algorithm, that calculates polarized radiative transfer including multiple scattering, as described in [10]. The thus determined solution of aerosol parameters will then be improved by iterating through a linearized radiative transfer model, which additionally provides a solid error analysis, as described in [11].

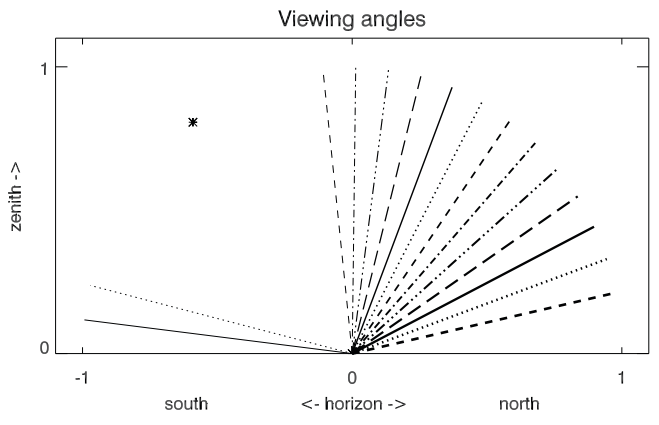

(a)

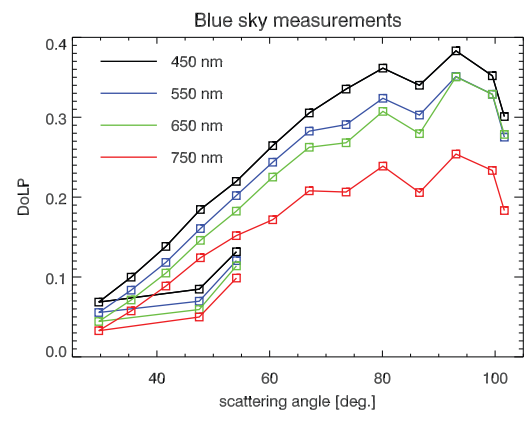

(b)

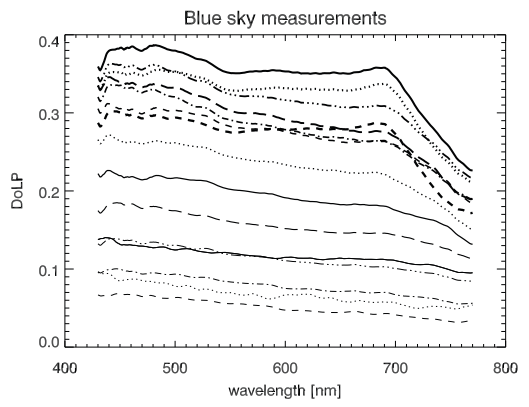

(c)

Figure 9: Multi-angle spectropolarimetric measurements of a blue sky, performed with the SPEX prototype. (a) The 15 viewing angles in the north-south plane. The approximate position of the Sun is depicted by the asterisk (the Sun is at an angle of $35^{\circ}$ with respect to the north-south plane). (b) The degree of polarization behavior with scattering angle, for different wavelengths. (c) The complete dataset, viz. the spectral degree of polarization for the different viewing angles. The coding of the viewing angles corresponds to the coding in subfigure (a). 


\section{OUTLOOK}

Our main present objective is to complete the characterization and calibration of the prototype instrument. Absolute calibration of the DoLP is required at levels other than $100 \%$ polarized light to determine absolute accuracy and the threshold sensitivity that can be achieved. This will be followed by rework on the prototype to resolve identified discrepancies. Next, the instrument is to be validated and verified by having it airborne. These activities will be undertaken prior to, or in parallel with, preparations for a specific mission opportunity.

The SPEX instrument concept is tailored to missions where a spacecraft orbits a target planetary body. The following missions present target opportunities for SPEX as a payload on a platform in orbit around a planetary body.

Chinese Mars Orbiter. Recently, the Chinese Academy for Science and Technology (CAST), offered a flight opportunity on their 2015 Mars orbiter mission HX-1. For that mission, SPEX will focus on the characterization of atmospheric dust, water clouds and $\mathrm{CO}_{2}$ clouds. Deriving the essential properties of dust (typical size, shape, refractive index, optical thickness) will enable a study of the role of dust in the Martian climate. Questions to be addressed are for example how local dust storms develop into much larger ones. Selection of SPEX for this mission will ultimately depend on the ability to achieve a sufficient level of maturity in the next few years.

JUICE. The SPEX team is investigating the feasibility of the polarimetric measurement concept for the Jupiter Icy Moons Explorer (JUICE)*. SPEX will target clouds and haze structures in the Jovian atmosphere and probe surfaces of the Galilean moons during flybys, with a closer polarimetric look at the surface of Ganymede during the final, low orbit of the spacecraft around this moon. One of the technical challenges is the complexity of the mission profile, demanding compatibility of the instrument concept under a variety of observational and environmental conditions.

ISS. SPEX is also perfectly suited as a remote sensing instrument for Earth atmospheric research. The large uncertainty of radiative forcing by aerosol is considered to be one of the major issues in present day climate studies [1] and precludes reliable predictions of climate change. In a dedicated low Earth orbit, SPEX would deliver multi-angle, spectral polarization data that are key to derive the required aerosol parameters. The International Space Station may also serve as a stepping-stone platform for SPEX to demonstrate the remote sensing capabilities. SPEX will be proposed as an instrument in response to ESA's call for experiments for Climate studies from the ISS. For this purpose, achieving good coverage is essential and this requires an extension of the field-of-view of the SPEX design. These points are currently under study.

In addition to these space-based applications, SPEX will also be part of a ground-based aerosol monitoring network. This research, carried out for the Dutch National Institute for Public Health and the Environment (RIVM), aims at determining the health effects of air quality. To that end, the local atmospheric aerosol properties will be remotely sensed, which is cheaper, faster, more accurate, and yields more detailed aerosol information than the current filtering system. At the moment, one ground-based SPEX is being constructed, which will be operational before 2012, to investigate the ability to create an autonomous ground-based network of SPEX instruments.

\section{ACKNOWLEDGMENTS}

The design study for SPEX, the prototype construction and testing, and the JUICE preparations are supported by PEP grants from the Netherlands Space Office.

\section{References}

[1] Solomon, S. (Ed.), [603 Climate Change 2007: The Physical Science Basis, Contribution of 604 Working Group (WG) 1 to the Fourth Assessment Report of the International Panel on 605 Climate Change (IPCC) (AR4)], Cambridge University Press, New York (2007)

\footnotetext{
${ }^{*}$ The mission was formerly a NASA-ESA collaboration, under the name of Europa Jupiter System Mission. With the withdrawal of NASA from number of collaborative programs, ESA is reformulating the Cosmic Vision L-class missions.
} 
[2] Hasekamp, O. P. and Landgraf, J., "Retrieval of aerosol properties over land surfaces: capabilities of multiple-viewing-angle intensity and polarization measurements", Applied Optics 46, 3332-3344 (2007)

[3] Mishchenko, M. I. and Travis, L. D., "Satellite retrieval of aerosol properties over the ocean using polarization as well as intensity of reflected sunlight", Journal of Geophysical Research 102, 16989-17013 (1997)

[4] Snik, F. and Keller, C. U., "Astronomical Polarimetry" in: 'Planets, Stars \& Stellar Systems', Springer (2011)

[5] Snik, F., Karalidi, T. and Keller, C. U., "Spectral modulation for full linear polarimetry", Applied Optics 48, 1337-1346 (2009)

[6] Stam, D. M., Laan, E., Snik, F., Karalidi, T., Keller, C. U., ter Horst, R., Navarro, R., Aas, C., de Vries, J., Oomen, G. and Hoogeveen, R., "Polarimetry of Mars with SPEX, an Innovative Spectropolarimeter", Third International Workshop on The Mars Atmosphere: Modeling and Observations, LPI Contribution No. 1447, 9078 (2008)

[7] Snik, F., Rietjens, J. H. H., van Harten, G., Stam, D. M., Keller, C. U., Smit, J. M., Laan, E. C., Verlaan, A. L., ter Horst, R., Navarra, R., Wielinga, K., Moon, S. G. and Voors, R., "SPEX: The Spectropolarimeter for Planetary EXploration", Proc. SPIE 7731, 77311B (2010)

[8] Baldridge, A. M., Hook, S. J., Grove, C. I. and Rivera, G., "The ASTER spectral library version 2.0", Remote Sensing of Environment 113, 711-715 (2009)

[9] Aben, I., Helderman, F., Stam, D. M. and Stammes, P., "Spectral fine-structure in the polarization of skylight", Geophysical Research Letters 26, 591-594 (1999)

[10] Stam, D. M., de Haan, J. F., Hovenier, J. W. and Stammes, P., "Degree of linear polarization of light emerging from the cloudless atmosphere in the oxygen A band", Journal of Geophysical Research 104, 16843-16858 (1999)

[11] Hasekamp, O. P., Landgraf, J., "Linearization of vector radiative transfer with respect to aerosol properties and its use in satellite remote sensing", Journal of Geophysical Research 100, 4203 (2005) 